\title{
Flexoelectric Effect on Vibration of Piezoelectric Microbeams Based on a Modified Couple Stress Theory
}

\author{
Xingjia Li and Ying Luo \\ Faculty of Civil Engineering and Mechanics, Jiangsu University, Zhenjiang 212013, China \\ Correspondence should be addressed to Ying Luo; luoying@ujs.edu.cn
}

Received 12 October 2016; Accepted 12 February 2017; Published 16 March 2017

Academic Editor: Paulo B. Gonçalves

Copyright ( 2017 Xingjia Li and Ying Luo. This is an open access article distributed under the Creative Commons Attribution License, which permits unrestricted use, distribution, and reproduction in any medium, provided the original work is properly cited.

\begin{abstract}
A novel electric Gibbs function was proposed for the piezoelectric microbeams (PMBs) by employing a modified couple stress theory. Based on the new Gibbs function and the Euler-Bernoulli beam theory, the governing equations which incorporate the effects of couple stress, flexoelectricity, and piezoelectricity were derived for the mechanics of PMBs. The analysis of the effective bending rigidity shows the effects of size and flexoelectricity can greaten the stiffness of PMBs so that the natural frequency increases significantly compared with the Euler-Bernoulli beam, and then the mechanical and electrical properties of PMBs are enhanced compared to the classical beam. This study can guide the design of microscale piezoelectric/flexoelectric structures which may find potential applications in the microelectromechanical systems (MEMS).
\end{abstract}

\section{Introduction}

Piezoelectricity is a well-studied electromechanical coupling effect in which the mechanical strain leads to electric polarization in piezoelectric crystals, or vice versa. Due to the excellent electromechanical characteristics at microscale [1], piezoelectric based microstructures have found a wide range of applications in microtechnology, like microtransducers, microgenerators, microresonantors, and so forth [2, 3]. Particularly, these above microstructures are quasi onedimensional structures which can be efficiently characterized by simple Euler-Bernoulli beam theory. Flexoelectricity [4-7] is, however, the coupling between the mechanical strain gradient and the electric polarization, and it is a universal electromechanical mechanism in all insulators including piezoelectric materials [8-11]. Shen and $\mathrm{Hu}[12$, 13] introduced an electric Gibbs function to consider both of the piezoelectricity and flexoelectricity and derived the governing equations for dielectric materials. Flexoelectricity has also been found to be useful when it is used for sensing purpose $[14,15]$. Moreover, the flexoelectric structures are theoretically predicted to be more sensitive when scaled down to microdomains $[16,17]$, yet their work did not take the size effect at microscale into account.
At microscale, size effect may be significant and even dominate the behavior of structures. Several strain gradient theories were dedicated to investigating the size effect [1821]. Among these work, Yang et al. [20] developed a modified couple stress theory with only one high-order material constant to account for the size effect on the microscale structures which makes the size effect more convenient to express. Afterwards, many efforts were devoted to studying the size effect on the mechanical behaviors of microbeams using the modified couple stress theory [22-25]. Among these, Park and Gao [22] developed a new model for the bending of a Bernoulli-Euler beam. Ma et al. [23] employed the Timoshenko beam model to study the size-dependence of static bending and vibration behaviors. Reddy [24] modified Euler-Bernoulli and Timoshenko models for functionally graded beams and studied the size-dependence of the deflection, vibration, and buckling behaviors for beam models. In these studies, the modified couple stress theory was used for elastic beams whereas the electromechanical coupling effects, for example, piezoelectric effect and flexoelectric effect, were not considered. Recently, the modified couple stress theory was utilized by Ansari et al. [25] for discussing the vibration characteristics of microbeams with piezoelectricity; however, flexoelectricity was not taken into account. Li et al. [26] 


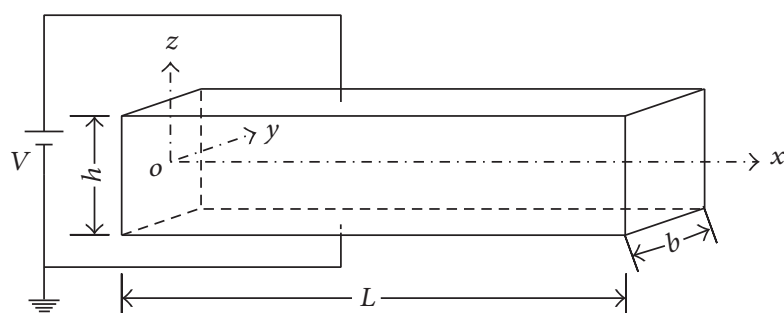

Figure 1: The schematic of the PMB whose cross section is a rectangle with width $b$ and height $h$. The origin of $x, y$, and $z$ coordinate system is located at the geometric centre of the left end. Herein an electric field is applied between the top $(z=h / 2)$ and bottom $(z=-h / 2)$ surfaces. The bottom surface is grounded.

reformulated a flexoelectric theory to study the bending behavior of PMBs with flexoelectricity. However, the vibration behaviors of PMBs with flexoelectricity have not been reported to date, which may be as important as the bending behavior in MEMS.

The goal of this paper is to study the natural frequency of PMBs with considering the size effect, piezoelectricity, and flexoelectricity. To achieve this goal, together with the Euler-Bernoulli beam theory, a new Gibbs function needs to be defined by employing a modified couple stress theory to derive the governing equations for the PMBs. The proposed study can be fundamental for the vibration behaviors of PMBs which may have potential application in the microelectromechanical systems (MEMS).

This paper is organized as follows. After introduction, Section 2 presents an electric Gibbs function based on the flexoelectricity theory and the modified couple stress theory. The dynamics equation and boundary conditions of piezoelectric beams were then derived by using the new Gibbs function and the Euler-Bernoulli beam model. Afterwards, the first-order natural frequencies under different boundary conditions of the current model and the traditional EulerBernoulli model were displayed. In particular, the natural frequency of simply supported PMBs subjected to a voltage across the thickness is solved as an example. Then the relationship between the frequency and the effective bending stiffness was discussed and employed to study the effects of couple stress, piezoelectricity, and flexoelectricity in Section 3. The new findings were summarized in Section 4.

\section{Dynamics Equation of Piezoelectric Microbeams with Flexoelectricity}

An Euler-Bernoulli beam in Figure 1 is considered:

$$
\begin{aligned}
& u_{x}=-\frac{\partial w(x, t)}{\partial x} z, \\
& u_{y}=0, \\
& u_{z}=w(x, t) .
\end{aligned}
$$

To account for the piezoelectric effect, flexoelectric effect, and nonlocal mechanical and electrical effects, an electric Gibbs energy density $G_{b}$ including the energy of elasticity, piezoelectricity, flexoelectricity, and couple stress was proposed by

$$
\begin{aligned}
G_{b}= & -\frac{1}{2}\left(k_{i j} E_{i} E_{j}+b_{i j k l} E_{i, j} E_{k, l}-C_{i j k l} \varepsilon_{i j} \varepsilon_{k l}\right) \\
& -e_{i j k} E_{i} \varepsilon_{j k}-\mu_{i j k l}\left(E_{k} \varepsilon_{i j, l}-\varepsilon_{i j} E_{k, l}\right)+\frac{1}{2} m_{i j} \chi_{i j},
\end{aligned}
$$

where $k_{i j}$ is dielectric constant tensor, $E_{i}$ and $E_{j}$ are electric field vectors, $b_{i j k l}$ is the nonlocal electrical coupling coefficient tensor, $C_{i j k l}$ is elastic stiffness tensor, $\varepsilon_{k l}$ is strain tensor, $e_{i j k}$ is piezoelectric coefficient tensor, $\mu_{i j k l}$ is a fourth-order tensor of the flexoelectric coefficient, and $\varepsilon_{k l, j}$ and $E_{k, l}$ are the gradients of strain and electric field. $m_{i j}$ is deviator part of couple stress tensor and $\chi_{i j}$ is the symmetric part of curvature tensor, which, using the modified couple stress theory [20, 22], are defined as

$$
\begin{aligned}
m_{i j} & =2 l^{2} \mu \chi_{i j}, \\
\chi_{i j} & =\frac{1}{2}\left(\theta_{i, j}+\theta_{j, i}\right), \\
\theta_{i} & =\frac{1}{2} e_{i j k} u_{k, l},
\end{aligned}
$$

where $\mu$ is Lamé's constants, $l$ is the material length scale parameter, $\theta_{i}$ is the rotation vector, and $u_{i}$ is the gradient of displacement vector, respectively.

Thus the constitutive equations for piezoelectric EulerBernoulli beams can be derived as

$$
\begin{aligned}
\sigma_{x x} & =\frac{\partial G_{b}}{\partial \varepsilon_{x x}}=C_{11} \varepsilon_{x x}-e_{31} E_{z}+\mu_{31} E_{z, z}, \\
\tau_{x x z} & =\frac{\partial G_{b}}{\partial \varepsilon_{x x, z}}=-\mu_{31} E_{z}, \\
m_{x y} & =\frac{\partial G_{b}}{\partial x_{x y}}=2 l^{2} \mu \chi_{k l}, \\
D_{z} & =\frac{\partial G_{b}}{\partial E_{z}}=k_{33} E_{z}+e_{31} \varepsilon_{x x}+\mu_{31} \varepsilon_{x x, z}, \\
Q_{z z} & =\frac{\partial G_{b}}{\partial E_{z, z}}=b_{33} E_{z, z}-\mu_{31} \varepsilon_{x x},
\end{aligned}
$$

where $\tau_{x x z}, D_{z}$, and $Q_{z z}$ denote the higher-order stress tensor, electric displacement vector, and electric quadrupole, respectively. In the absence of free charges, Gauss's law requires

$$
-\frac{\partial^{2} Q_{z z}}{\partial z^{2}}+\frac{\partial D_{z}}{\partial z}=0
$$

Substituting (4) into (5) leads to

$$
k_{33} E_{z, z}+e_{31} \varepsilon_{x x, z}+\mu_{31} \varepsilon_{x x, z z}-b_{33} E_{z, z z z}=0 .
$$

The electrical boundary conditions are given by

$$
\begin{gathered}
\Phi\left(-\frac{h}{2}\right)=0, \\
\Phi\left(\frac{h}{2}\right)=V,
\end{gathered}
$$


where $\Phi$ is the electric potential. Combining $E_{z}=-\partial \Phi / \partial z$ with (4)-(7) yields

$$
\begin{aligned}
\Phi= & \frac{V}{2}+\frac{V}{h} z+\frac{e_{31}}{2 k_{33}}\left(\frac{h^{2}}{4}-z^{2}\right)\left(\frac{\partial^{2} w}{\partial x^{2}}\right) \\
& -\frac{\mu_{31}}{2 k_{33}}\left(\frac{\partial^{2} w}{\partial x^{2}}\right) z+\frac{e_{31}}{\eta^{2} k_{33}} \frac{e^{\eta z}+e^{-\eta z}}{e^{\eta h / 2}+e^{-\eta h / 2}}\left(\frac{\partial^{2} w}{\partial x^{2}}\right) \\
& +\frac{\mu_{31} h}{4 \eta^{2} k_{33}} \frac{e^{\eta z}-e^{-\eta z}}{e^{\eta h / 2}-e^{-\eta h / 2}} \frac{e^{\eta z}+e^{-\eta z}}{e^{\eta h / 2}+e^{-\eta h / 2}}
\end{aligned}
$$

with $\eta=\sqrt{k_{33} / b_{33}}$. Neglecting the high-order nonlocal electric effect in (8) by assuming $b_{33}=0$ yields the following equations $[27,28]$ :

$$
\begin{aligned}
\Phi= & \frac{V}{2}+\frac{V}{h} z+\frac{e_{31}}{2 k_{33}}\left(\frac{h^{2}}{4}-z^{2}\right)\left(\frac{\partial^{2} w}{\partial x^{2}}\right) \\
& -\frac{\mu_{31}}{2 k_{33}}\left(\frac{\partial^{2} w}{\partial x^{2}}\right) z \\
E_{z}= & \left(\frac{e_{31}}{k_{33}} z-\frac{\mu_{31}}{2 k_{33}}\right) \frac{\partial^{2} w}{\partial x^{2}}-\frac{V}{h}, \\
E_{z, z}= & \frac{e_{31}}{k_{33}}\left(\frac{\partial^{2} w}{\partial x^{2}}\right), \\
\sigma_{x x}= & -C_{11}\left(\frac{\partial^{2} w}{\partial x^{2}}\right) z-\frac{e_{31}^{2}}{k_{33}}\left(\frac{\partial^{2} w}{\partial x^{2}}\right) z \\
& +\frac{e_{31} \mu_{31}}{2 k_{33}}\left(\frac{\partial^{2} w}{\partial x^{2}}\right)+e_{31} \frac{V}{h}, \\
\tau_{x x z}= & -\frac{e_{31} \mu_{31}}{k_{33}}\left(\frac{\partial^{2} w}{\partial x^{2}}\right) z-\frac{\mu_{31}^{2}}{2 k_{33}}\left(\frac{\partial^{2} w}{\partial x^{2}}\right)+\frac{\mu_{31} V}{h} .
\end{aligned}
$$

The elastic energy $U$, kinetic energy $T$, and external work $W$ are calculated by the following equations:

$$
\begin{aligned}
& U=\frac{1}{2} \int_{0}^{l} \int_{A}\left(\sigma_{x x} \varepsilon_{x x}+2 m_{x y} \chi_{x y}+\tau_{x x z} \varepsilon_{x x, z}\right) d A d x, \\
& T=\frac{1}{2} \int_{0}^{l} \int_{A} \rho\left(\frac{\partial u_{z}}{\partial t}\right) d A d x, \\
& W=-\frac{1}{2} \int_{0}^{l} N_{x x}\left(\frac{\partial w}{\partial x}\right)^{2} d x, \\
& N_{x x}=\int_{A} \sigma_{x x} d A .
\end{aligned}
$$

Hamilton's principle reads

$$
\int_{t_{1}}^{t_{2}}(\delta T-\delta U+\delta W) d t=0 .
$$

Then the governing equation for their transverse vibration can be derived by the vibrational principle

$$
-\rho A \frac{\partial^{2} w}{\partial t^{2}}=(E I)_{\mathrm{eff}} \frac{\partial^{4} w}{\partial x^{4}}-P_{\mathrm{eff}} \frac{\partial^{2} w}{\partial x^{2}},
$$

where

$$
\begin{aligned}
(E I)_{\mathrm{eff}} & =C_{11} I+\frac{e_{31}^{2} I}{k_{33}}+A \mu l^{2}+\frac{\mu_{31}^{2} A}{2 k_{33}}, \\
P_{\mathrm{eff}} & =e_{31} V b .
\end{aligned}
$$

For facilitating, make the substitution of $(E I)_{e}=C_{11} I$, $(E I)_{p}=e_{31}^{2} I / k_{33},(E I)_{c}=A \mu l^{2}$, and $(E I)_{f}=\mu_{31}^{2} A / 2 k_{33}$, and then we have

$$
\begin{aligned}
(E I)_{\mathrm{eff}} & =(E I)_{e}+(E I)_{p}+(E I)_{c}+(E I)_{f}, \\
P_{\mathrm{eff}} & =e_{31} V b .
\end{aligned}
$$

If the flexoelectric effect is neglected, the effective bending rigidity can be reduced to

$$
\begin{aligned}
(E I)_{\mathrm{eff}}^{p} & =(\lambda+2 \mu) I+\frac{e_{31}^{2} I}{k_{33}}+A \mu l^{2}, \\
P_{\mathrm{eff}}^{p} & =e_{31} V b .
\end{aligned}
$$

If the working temperature of PMBs is higher than Curie's point, the piezoelectric effect will disappear and then the effective bending rigidity can be rewritten as

$$
\begin{aligned}
(E I)_{\mathrm{eff}}^{f} & =(\lambda+2 \mu) I+\frac{\mu_{31}^{2} A}{2 k_{33}}+A \mu l^{2}, \\
P_{\mathrm{eff}}^{f} & =0 .
\end{aligned}
$$

The geometric parameters are defined as

$$
\int_{A}\left(1, z, z^{2}\right) d A=(A, 0, I)
$$

with $A$ and $I$ being the cross section area and inertia moment of beams. The natural frequencies for PMBs with different boundary conditions, such as simply supported (S$\mathrm{S})$, cantilever (C-F), and clamped-clamped (C-C) types, can be calculated based on the following characteristic equations:

$$
\begin{aligned}
& \sin \left(s_{2} L\right)=0 \quad(\mathrm{~S}-\mathrm{S}), \\
& s_{1}^{4}+s_{2}^{4}+s_{1} s_{2}\left(s_{2}^{2}-s_{1}^{2}\right) \sinh \left(s_{1} L\right) \sin \left(s_{2} L\right) \\
& \quad+2 s_{1}^{2} s_{2}^{2} \cosh \left(s_{1} L\right) \cos \left(s_{2} L\right)=0 \quad(\mathrm{C}-\mathrm{F}), \\
& 2 s_{1} s_{2}-2 s_{1} s_{2} \cosh \left(s_{1} L\right) \cos \left(s_{2} L\right) \\
& \quad+\left(s_{1}^{2}-s_{2}^{2}\right) \sinh \left(s_{1} L\right) \sin \left(s_{2} L\right)=0 \quad(\mathrm{C}-\mathrm{C}),
\end{aligned}
$$

where

$$
\begin{aligned}
& s_{1}=\left(\frac{P_{\mathrm{eff}}+\sqrt{P_{\mathrm{eff}}^{2}+4 \rho A \omega^{2}(E I)_{\mathrm{eff}}}}{2(E I)_{\mathrm{eff}}}\right)^{1 / 2}, \\
& s_{2}=\left(\frac{-P_{\mathrm{eff}}+\sqrt{P_{\mathrm{eff}}^{2}+4 \rho A \omega^{2}(E I)_{\mathrm{eff}}}}{2(E I)_{\mathrm{eff}}}\right)^{1 / 2}
\end{aligned}
$$

with $\omega$ representing the angular resonant frequency. 


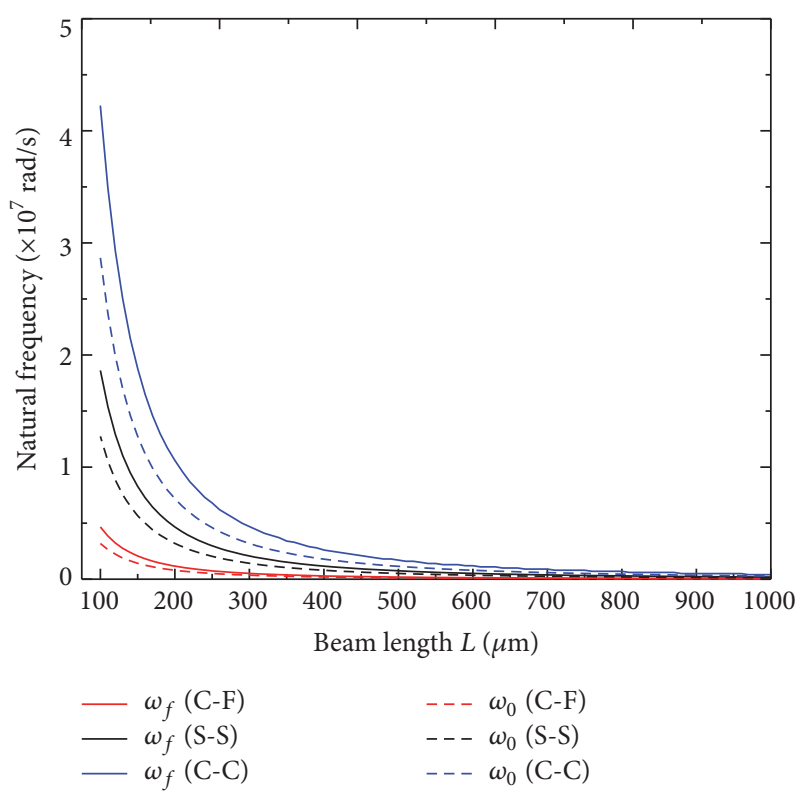

FIGURE 2: The first natural frequency of the present beam model $\left(\omega_{f}\right)$ without voltage and the traditional Euler-Bernoulli beam model $\left(\omega_{0}\right)$ versus beam length with different boundary conditions.

TABLE 1: Material constants of the piezoelectric microbeam [8-11].

\begin{tabular}{lccccc}
\hline$E$ & $v$ & $\rho$ & $e_{31}$ & $\mu_{31}$ & $\varepsilon_{r}$ \\
\hline $150 \mathrm{GPa}$ & 0.33 & $7.5 \times 10^{3} \mathrm{Kg} / \mathrm{m}^{3}$ & $-4.35 \mathrm{C} / \mathrm{m}^{2}$ & $10 \mu \mathrm{C} / \mathrm{m}$ & 2300 \\
\hline
\end{tabular}

$E$ is Young's modulus, $\nu$ is Poisson's ratio, $\rho$ is mass density, $e_{31}$ is piezoelectric constant, $\mu_{31}$ is flexoelectric constant, $\varepsilon_{r}$ is relative dielectric constant, and the vacuum dielectric constant $\varepsilon_{0}=8.85 \times 10^{-12} \mathrm{~F} / \mathrm{m}$.

\section{Results and Discussion}

In this section the formulae derived in Section 2 will be used to study the fundamental vibration of the PMBs. The emphasis is on the effects of size, piezoelectricity, and flexoelectricity. To this end, $\mathrm{BaTiO}_{3}$ was chosen as the material of the PMBs where the material constants are listed in Table 1 . The cross section of $\mathrm{BaTiO}_{3}$ beam is assumed to be square; that is, $b=h$.

Employing (13), (18), and (19) and the material constants in Table 1, we can easily get the first-order natural frequency of the PMBs $\omega_{f}$. Moreover, the first natural frequency of traditional Euler-Bernoulli beam model $\omega_{0}=(i \pi / L)^{2} \sqrt{E I / \rho A}$ ( $i=0.5$ for C-F type, $i=1$ for S-S type, and $i=1.5$ for C-C type) is employed for comparison. Here $\omega_{f}$ denotes the natural frequency of the present model; that is, both effects of piezoelectricity and flexoelectricity are taken into consideration. Correspondingly, $\omega_{p}$ represents the natural frequency when only piezoelectric effect is considered. The difference between the present model and the traditional model can be seen in Figure 2. Hereby PMBs have $h=10 \mu \mathrm{m}$ and $l=5 \mu \mathrm{m}$.

From Figure 2 we can see that the differences in the frequencies between the present model and the traditional model increase significantly when the beam length decreases to less than $400 \mu \mathrm{m}$ and then turn to be nearly the same when the beam length increases to more than $800 \mu \mathrm{m}$. In general, the trends in the frequencies of the present model resemble that of the traditional model because the frequency equation of $\left.\omega_{f}\right|_{V=0}=(i \pi / L)^{2} \sqrt{(E I)_{\mathrm{eff}} / \rho A}$ and $\omega_{0}$ are very alike with small difference between the bending stiffness $(E I)_{\mathrm{eff}}$ and $(E I)$.

3.1. Effect of Piezoelectricity and Flexoelectricity. Throughout the following sections of this work we will calculate the first normalized natural frequencies $\bar{\omega}_{p}=\omega_{p} / \omega_{0}$ and $\bar{\omega}_{f}=\omega_{f} / \omega_{0}$, and, for facilitating the discussion, the simply supported (S-S) boundary condition was chosen as a representative. The normalized frequencies are shown in Figure 3 for PMBs where length-to-thickness ratio $L / h$ is fixed at 20 and the length $L$ increases from $20 \mu \mathrm{m}$ to $1000 \mu \mathrm{m}$. Accordingly the thickness $h$ grows from $1 \mu \mathrm{m}$ to $50 \mu \mathrm{m}$ and the material length scale parameter $l$ is defined by $l=h / 2$ as others do in $[25,26]$ which increases from $0.5 \mu \mathrm{m}$ to $25 \mu \mathrm{m}$. In addition, the voltage $V=-2 \mathrm{~V}, 0$, and $2 \mathrm{~V}$ are applied to reveal the effects of piezoelectricity and flexoelectricity.

In Figure 3(a), where only piezoelectric effect is considered (i.e., without flexoelectricity), $\bar{\omega}_{p}=1.461$ is obtained at $V=0$ showing that the size effect increases by $46.1 \%$ for all PMBs of different length $L$ and length scale parameter $l$. Equations (13) and (14) show the contribution of the size effect $(E I)_{c}$ on the effective bending stiffness $(E I)_{\text {eff }}$ of the PMBs. At $L=20 \mu \mathrm{m}, \bar{\omega}_{p}$ increases to 1.465 when $V=-2 \mathrm{~V}$ is applied whereas it deceases to 1.457 at $V=2 \mathrm{~V}$. This result shows that the external voltage leads to the increase (or decrease) of the frequency of PMBs in the presence of a negative (or positive) voltage. A $4 \mathrm{~V}$ external voltage can tune the frequency of PMBs by $0.55 \%$, and the tuning of frequency can be greater under greater external voltage. Such a piezoelectric effect can be explained by the electromechanical coupling term $(E I)_{p}$ in $(E I)_{\text {eff }}$ and the extra-axial force $P_{\text {eff }}$ in (13) and (14). Specifically, such a piezoelectric effect decreases monotonically with rising length $L$ and shows significant length-dependence. As a result $\bar{\omega}_{p}$ with $V=-2 \mathrm{~V}$ or $2 \mathrm{~V}$ approaches $\bar{\omega}_{f}=1.461$ when the length $L$ is sufficiently large; for example, $L>900 \mu \mathrm{m}$. In this case the piezoelectric effect turns out to be negligible.

Different from Figure 3(a), both effects of piezoelectricity and flexoelectricity are considered in Figure 3(b) for the normalized frequency $\bar{\omega}_{f}$. Thus the difference between the results in Figures 3(a) and 3(b) [i.e., $\left(\bar{\omega}_{f}-\bar{\omega}_{p}\right)$ ] reveals the effect of flexoelectricity on the normalized fundamental frequency. First it is noted in Figure 3(b) that, at $V=0$, $\bar{\omega}_{f}$ becomes length-dependent, which is in contrast to the length-independent $\bar{\omega}_{p}$ in Figure 3(a). At $L=20 \mu \mathrm{m}$ flexoelectric effect slightly increases the normalized frequency by $0.73 \%$ relative to $\bar{\omega}_{p}$. The relative increment then decreases with rising $L$ and becomes negligible for long and thick PMBs. Qualitatively similar trend is observed for the flexoelectric effect at $V=-2 \mathrm{~V}$, which raises the normalized frequency by $0.26 \%$ at $L=20 \mu \mathrm{m}$. The increase of the frequency decreases with rising $L$ and almost vanishes at $L=1000 \mu \mathrm{m}$. On the other hand, when positive voltage $2 \mathrm{~V}$ is applied, it is 


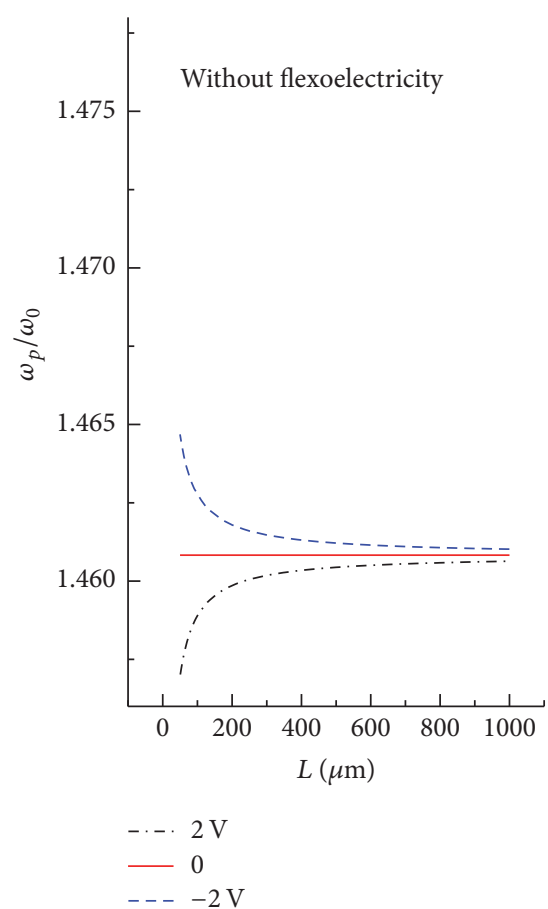

(a)

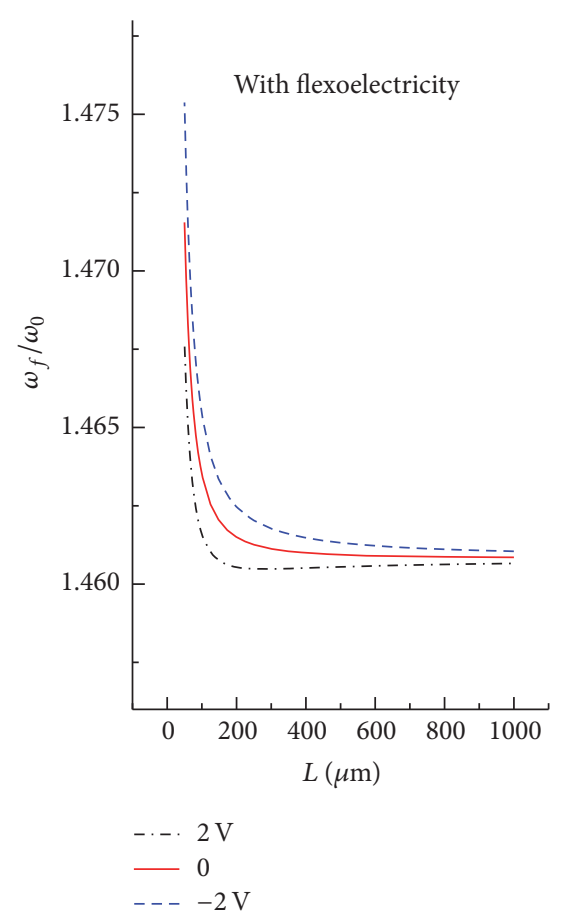

(b)

FIGURE 3: Length-dependence of the normalized fundamental frequency calculated for (a) $\omega_{p} / \omega_{0}$ of PMBs with only piezoelectric effect and (b) $\omega_{f} / \omega_{0}$ with both effects of piezoelectricity and flexoelectricity.

noted in Figure 3(b) that $\bar{\omega}_{f}$ first decreases with rising $L$ and reaches the lowest value at $L=302 \mu \mathrm{m}$. It then increases with increase of $L$ and reaches close to $\bar{\omega}_{f}=1.461$ when the length is greater than $900 \mu \mathrm{m}$. This behavior of $\bar{\omega}_{f}$ at $2 \mathrm{~V}$ is qualitatively different from the corresponding $L$-dependence of $\bar{\omega}_{p}$ in Figure 3(a) and can be attributed to the competition between the reversed effects of piezoelectricity (with positive voltage) and the flexoelectricity on the normalized frequency. Here it is noted that considering flexoelectricity always leads to the increase of the frequency independent of the voltage applied. This behavior can be attributed to the fact that flexoelectricity influences the frequency via the electromechanical coupling term $(E I)_{f}$ in $(E I)_{\text {eff }}$ found in (14), which always greatens the effective bending stiffness and also makes the natural frequency size-dependent. Though it can be seen from Figure 3(a) the positive voltage decreases the natural frequency with decreasing $L$, when $L$ is shorter than $302 \mu \mathrm{m}$ flexoelectric effect dominates the electromechanical behaviors resulting in a significant rise in the natural frequency.

\subsection{Size Effect on the Electromechanical Coupling in Vibration.} After investigating the effects of piezoelectricity and flexoelectricity, we would like to further reveal the influence of the size effect of piezoelectricity and flexoelectricity. Here we first calculated the $l$-dependence of $\bar{\omega}_{p}$ (without flexoelectricity) in Figure 4 for PMBs subjected to a voltage of $-2 \mathrm{~V}, 0$, and $2 \mathrm{~V}$, respectively. In the calculations two groups of PMBs are considered, which have an aspect ratio $L / h=20$ and 40 , respectively. In addition the scale material constant $l$ is

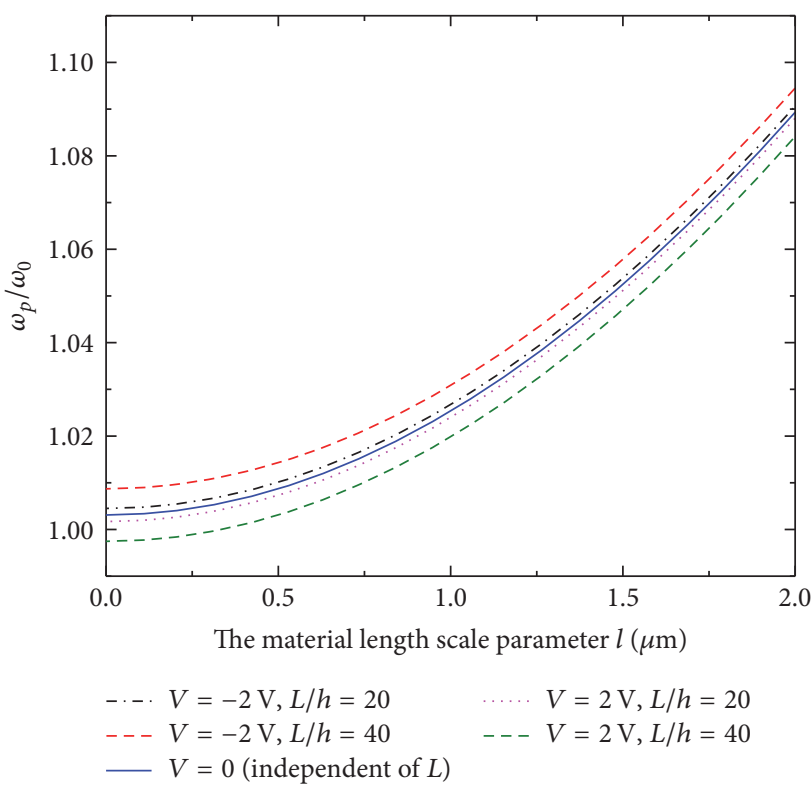

Figure 4: $l$-dependence of $\omega_{p} / \omega_{0}$ obtained for PMBs where the effect of piezoelectricity is considered but the effect of flexoelectricity is ignored. Here PMBs have $h=10 \mu \mathrm{m}$ and $L / h=20$ and 40, respectively.

assumed to be a constant and increases from 0 (classical theory) to $2 \mu \mathrm{m}$. Figure 4 shows that in general $\bar{\omega}_{p}$ increases with growing $l$ for all the PMBs under a given voltage. It is 


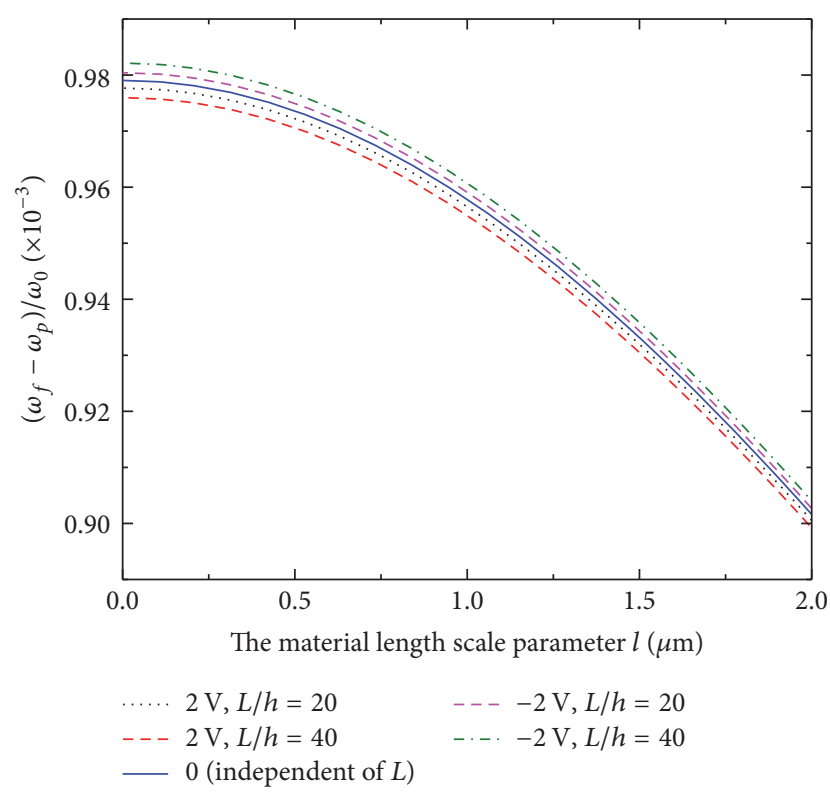

FIGURE 5: $l$-dependence of $\left(\omega_{f}-\omega_{p}\right) / \omega_{0}$ obtained for PMBs where $h$ is fixed at $10 \mu \mathrm{m}$ and $L / h$ is equal to 20 and 40 , respectively.

worth mentioning that at $V=0$ the results remain unchanged no matter what aspect ratio $L / h$ is considered. Specifically, the curves associated with different nonzero voltages are almost parallel with one another. These results indicate that the change in normalized frequency, that is, $\left(\left.\bar{\omega}_{p}\right|_{V \neq 0}-\left.\bar{\omega}_{p}\right|_{V=0}\right)$ due to piezoelectric effect, is not very sensitive to the variation of the scale parameter $l$ (the subscript shows the voltage applied). On the other hand, as $\left.\bar{\omega}_{p}\right|_{V=0}$ rises with rising $l$, the relative change $\left(\left.\bar{\omega}_{p}\right|_{V \neq 0}-\left.\bar{\omega}_{p}\right|_{V=0}\right) /\left.\bar{\omega}_{p}\right|_{V=0}$ due to piezoelectric effect decreases significantly with increasing $l$ (the subscript shows the voltage considered). For example, at $l=0$ relative change due to $V=-2 \mathrm{~V}(V=-2 \mathrm{~V})$ is $0.56 \%(-0.56 \%)$ for PMBs with $L=40 \mathrm{~h}$. However, at $l=2 \mu \mathrm{m}$ such a relative change decreases to $0.47 \%$ at $V=-2 \mathrm{~V}$ and $-0.48 \%$ at $V=$ $2 \mathrm{~V}$. In addition it is also noticed that for a constant $l$ the piezoelectric effect is less pronounced for shorter PMBs with greater $L / h=20$.

Next we shall further exam the influence of the size effect on the effect of flexoelectricity by calculating the $l$ dependence of $\bar{\omega}_{f-p}=\bar{\omega}_{f}-\bar{\omega}_{p}$ in Figure 5. It is easy to understand that $\omega_{f-p}$ reflects the pure effect of flexoelectricity. Figure 5 shows that, at $V=0, \bar{\omega}_{f-p}$ decreases from around $0.98 \times 10^{-3}$ to $0.90 \times 10^{-3}$ when $l$ increases from 0 to $2.0 \mu \mathrm{m}$. These results indicate that the flexoelectricity increases the frequency of PMBs by less than $0.1 \%$, and such an effect is found to be even smaller for greater $l$ or larger size effect. In addition, at $V=-2 \mathrm{~V}$ (or $2 \mathrm{~V}$ ) the effect of flexoelectricity on PMB increases (decreases) slightly but the influence of voltage becomes smaller for greater $l$. Indeed it is seen from Figure 5 that, in terms of $L / h=40$ when $l=0,\left.\bar{\omega}_{f-p}\right|_{V=-2 \mathrm{~V}}$ is $0.32 \%$ higher than $\left.\bar{\omega}_{f-p}\right|_{V=0}$ but it declines to $0.27 \%$ when $l=2 \mu \mathrm{m}$. In other words, by increasing $l$ the effective bending stiffness by flexoelectricity increases; nevertheless the frequency difference of $\bar{\omega}_{f-p}$ between existing external voltage and no external voltage diminishes.

\section{Conclusions}

A novel electric Gibbs function was proposed to describe the size effect on the electromechanical coupling behaviors of $\mathrm{PMBs}$ by incorporating a modified couple stress theory into Euler-Bernoulli beam model. Based on the obtained model, the effects of piezoelectricity and flexoelectricity and the size effect were examined for the vibration behavior of PMBs. It is found that, at the microscale, the size effect can raise the effective stiffness of the structure and thus increases the natural frequency. With the assumed value of the material length scale parameter $l$, the couple stress dominates the mechanical behavior of the PMBs and increases with rising $l$. Piezoelectric effect is achieved, which can increase or decrease the frequency depending on the axial force induced by external voltage. In particular, the contribution of piezoelectric effect to the natural frequency was found to be decreasing with rising $l$ or geometric size of PMBs. In the meantime, the flexoelectricity can also raise the structural stiffness via an electromechanical coupling term, which is relatively small but detectable and always increases the frequency of PMBs. With the development of material science, the higher performance of flexoelectric materials might play an more important role in MEMS.

\section{Conflicts of Interest}

The authors declare that there are no conflicts of interest regarding the publication of this paper.

\section{Acknowledgments}

The research reported herein was supported under NSFC (no. 11272138) and Specialized Research Fund for the Doctoral Program of Higher Education of China (no. 20123227130002).

\section{References}

[1] E. S. Hung and S. D. Senturia, "Extending the travel range of analog-tuned electrostatic actuators," Journal of Microelectromechanical Systems, vol. 8, no. 4, pp. 497-505, 1999.

[2] X. Li, B. Bhushan, K. Takashima, C.-W. Baek, and Y.-K. Kim, "Mechanical characterization of micro/nanoscale structures for MEMS/NEMS applications using nanoindentation techniques," Ultramicroscopy, vol. 97, no. 1-4, pp. 481-494, 2003.

[3] Y. Moser and M. A. M. Gijs, "Miniaturized flexible temperature sensor," Journal of Microelectromechanical Systems, vol. 16, no. 6, pp. 1349-1354, 2007.

[4] A. K. Tagantsev, "Piezoelectricity and flexoelectricity in crystalline dielectrics," Physical Review B, vol. 34, no. 8, pp. 58835889, 1986.

[5] L. E. Cross, "Flexoelectric effects: charge separation in insulating solids subjected to elastic strain gradients," Journal of Materials Science, vol. 41, no. 1, pp. 53-63, 2006.

[6] P. Zubko, G. Catalan, A. Buckley, P. R. L. Welche, and J. F. Scott, "Strain-gradient-induced polarization in $\mathrm{SrTiO}_{3}$ single 
crystals," Physical Review Letters, vol. 99, no. 16, Article ID 167601, 4 pages, 2007.

[7] N. D. Sharma, R. Maranganti, and P. Sharma, "On the possibility of piezoelectric nanocomposites without using piezoelectric materials," Journal of the Mechanics and Physics of Solids, vol. 55, no. 11, pp. 2328-2350, 2007.

[8] W. Ma and L. E. Cross, "Observation of the flexoelectric effect in relaxor $\mathrm{Pb}(\mathrm{Mg} 1 / 3 \mathrm{Nb} 2 / 3) \mathrm{O} 3$ ceramics," Applied Physics Letters, vol. 78, no. 19, pp. 2920-2921, 2001.

[9] W. Ma and L. E. Cross, "Flexoelectric polarization of barium strontium titanate in the paraelectric state," Applied Physics Letters, vol. 81, no. 18, pp. 3440-3442, 2002.

[10] W. Ma and L. E. Cross, "Flexoelectric effect in ceramic lead zirconate titanate," Applied Physics Letters, vol. 86, no. 7, Article ID 072905, 2005.

[11] W. Ma and L. E. Cross, "Flexoelectricity of barium titanate," Applied Physics Letters, vol. 88, no. 23, Article ID 232902, 2006.

[12] S. Shen and S. Hu, "A theory of flexoelectricity with surface effect for elastic dielectrics," Journal of the Mechanics and Physics of Solids, vol. 58, no. 5, pp. 665-677, 2010.

[13] S. Hu and S. Shen, "Variational principles and governing equations in nano-dielectrics with the flexoelectric effect," Science China Physics, Mechanics and Astronomy, vol. 53, no. 8, pp. 1497-1504, 2010.

[14] X. Yan, W. Huang, S. Ryung Kwon, S. Yang, X. Jiang, and F.-G. Yuan, "A sensor for the direct measurement of curvature based on flexoelectricity," Smart Materials and Structures, vol. 22, no. 8, Article ID 085016, 2013.

[15] W. Huang, X. Yan, S. R. Kwon, S. Zhang, F.-G. Yuan, and X. Jiang, "Flexoelectric strain gradient detection using $\mathrm{Ba} 0.64 \mathrm{Sr}$ 0.36TiO3 for sensing," Applied Physics Letters, vol. 101, no. 25, Article ID 252903, 2012.

[16] S. R. Kwon, W. B. Huang, S. J. Zhang, F. G. Yuan, and X. N. Jiang, "Flexoelectric sensing using a multilayered barium strontium titanate structure," Smart Materials and Structures, vol. 22, no. 11, Article ID 115017, 2013.

[17] S. R. Kwon, W. Huang, L. Shu, F.-G. Yuan, J.-P. Maria, and X. Jiang, "Flexoelectricity in barium strontium titanate thin film," Applied Physics Letters, vol. 105, no. 14, Article ID 142904, 2014.

[18] R. D. Mindlin and N. N. Eshel, "On first strain-gradient theories in linear elasticity," International Journal of Solids and Structures, vol. 4, no. 1, pp. 109-124, 1968.

[19] R. A. Toupin, "Theories of elasticity with couple-stress," Archive for Rational Mechanics and Analysis, vol. 17, pp. 85-112, 1964.

[20] F. Yang, A. C. M. Chong, D. C. C. Lam, and P. Tong, "Couple stress based strain gradient theory for elasticity," International Journal of Solids and Structures, vol. 39, no. 10, pp. 2731-2743, 2002.

[21] D. C. C. Lam, F. Yang, A. C. M. Chong, J. Wang, and P. Tong, "Experiments and theory in strain gradient elasticity," Journal of the Mechanics and Physics of Solids, vol. 51, no. 8, pp. 14771508, 2003.

[22] S. K. Park and X.-L. Gao, "Bernoulli-Euler beam model based on a modified couple stress theory," Journal of Micromechanics and Microengineering, vol. 16, no. 11, pp. 2355-2359, 2006.

[23] H. M. Ma, X.-L. Gao, and J. N. Reddy, "A microstructuredependent Timoshenko beam model based on a modified couple stress theory," Journal of the Mechanics and Physics of Solids, vol. 56, no. 12, pp. 3379-3391, 2008.

[24] J. N. Reddy, "Microstructure-dependent couple stress theories of functionally graded beams," Journal of the Mechanics and Physics of Solids, vol. 59, no. 11, pp. 2382-2399, 2011.
[25] R. Ansari, M. A. Ashrafi, and S. Hosseinzadeh, "Vibration characteristics of piezoelectric microbeams based on the modified couple stress theory," Shock and Vibration, vol. 2014, Article ID 598292, 12 pages, 2014.

[26] A. Li, S. Zhou, L. Qi, and X. Chen, "A reformulated flexoelectric theory for isotropic dielectrics," Journal of Physics D: Applied Physics, vol. 48, no. 46, Article ID 465502, 2015.

[27] L. Shu, F. Li, W. Huang, X. Wei, X. Yao, and X. Jiang, "Relationship between direct and converse flexoelectric coefficients," Journal of Applied Physics, vol. 116, no. 14, Article ID 144105, 2014.

[28] X. Liang, S. Hu, and S. Shen, "Size-dependent buckling and vibration behaviors of piezoelectric nanostructures due to flexoelectricity," Smart Materials and Structures, vol. 24, no. 10, Article ID 105012, 2015. 


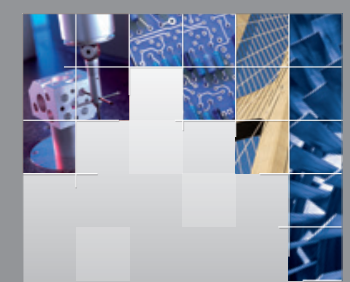

\section{Enfincering}
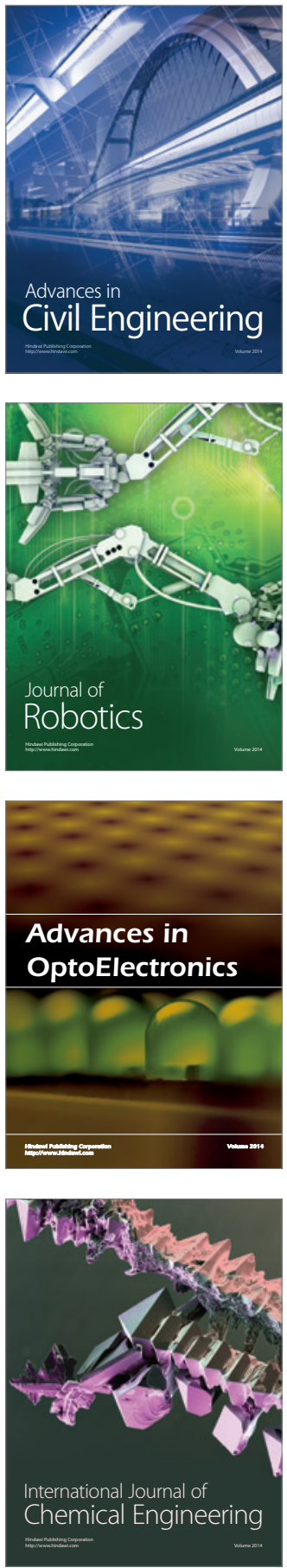

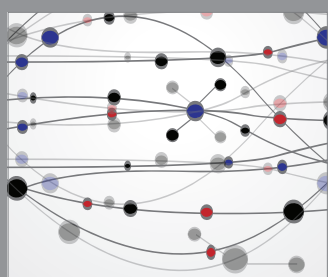

The Scientific World Journal

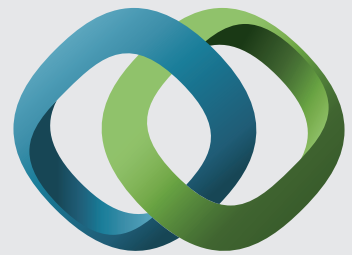

\section{Hindawi}

Submit your manuscripts at

https://www.hindawi.com
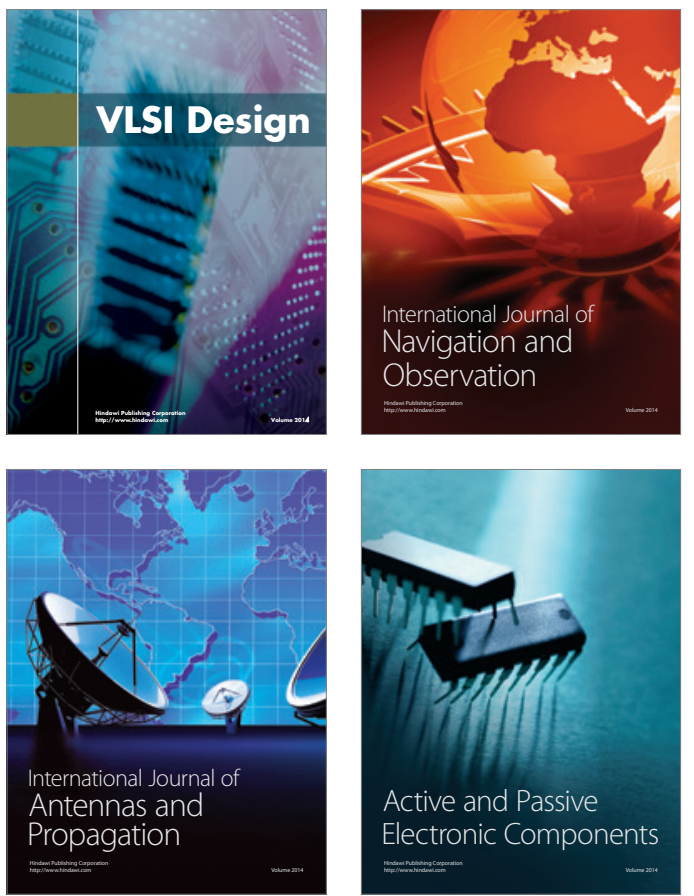
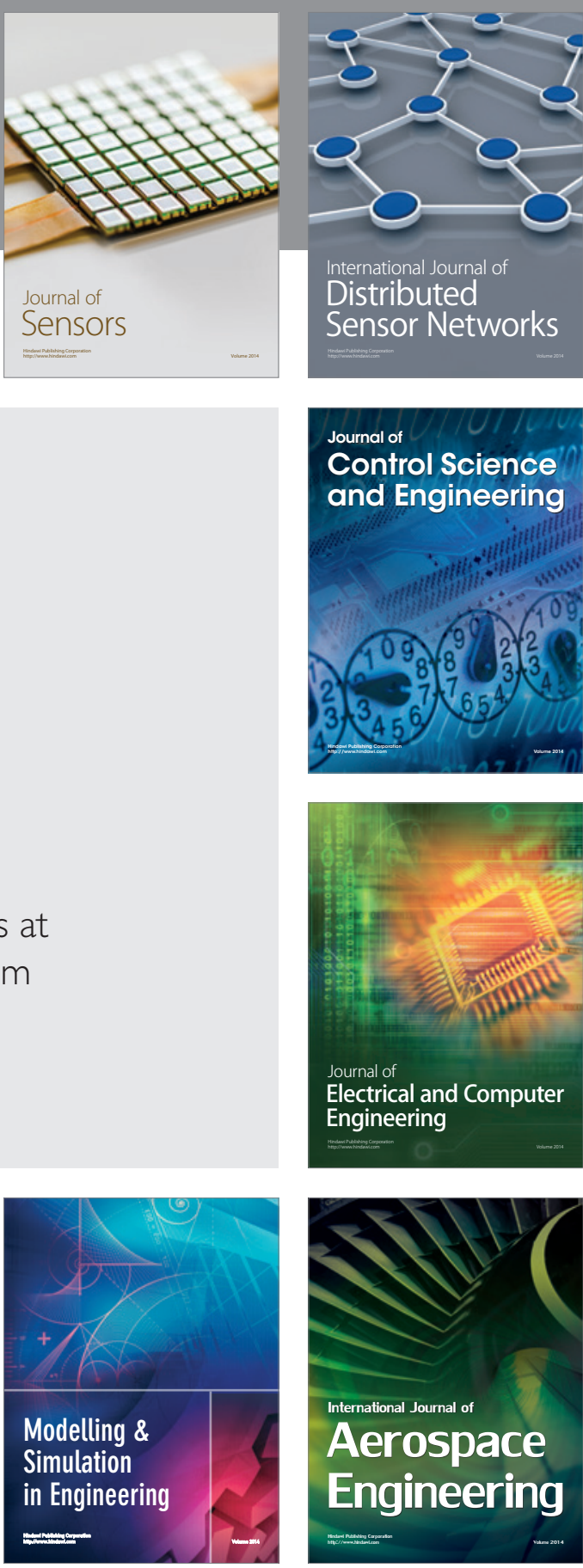

International Journal of

Distributed

Sensor Networks

$-$

Joumal of

Control Science

and Engineering
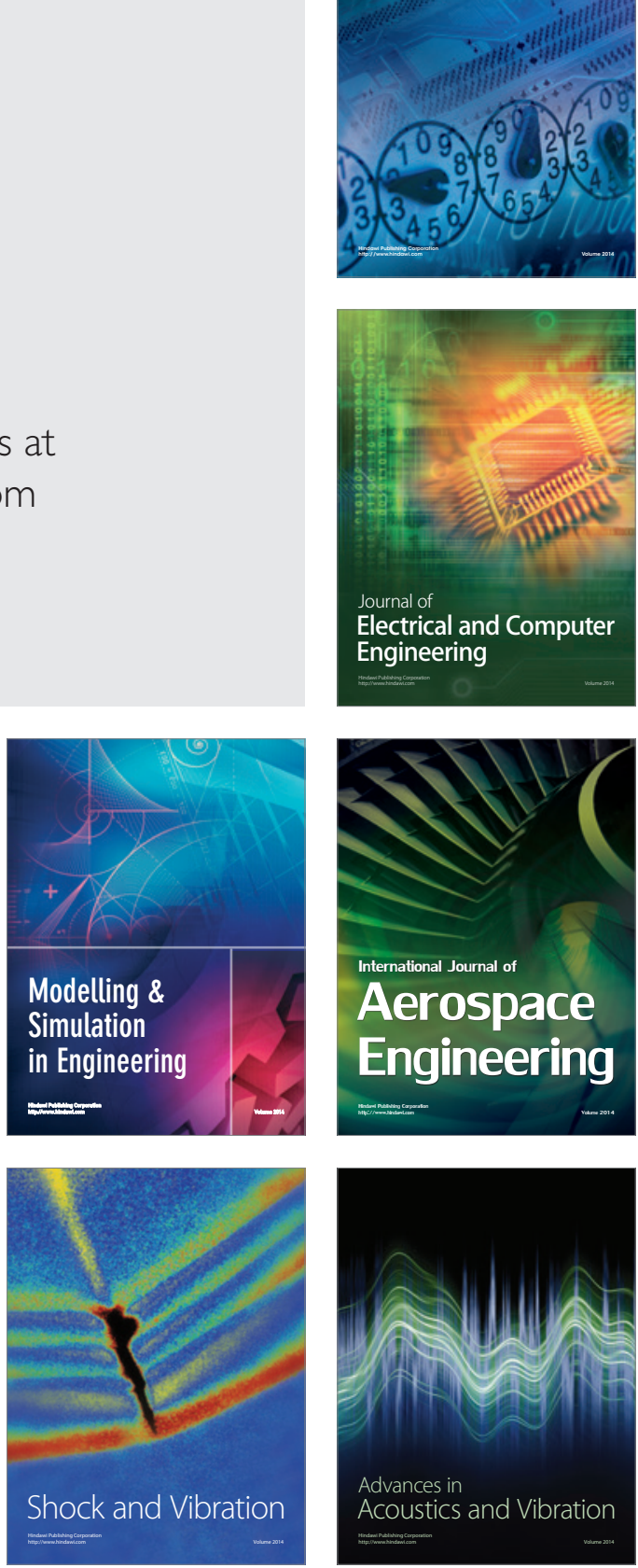